Published in Review of Progress in Quantitative Nondestructive Evaluation, Vol. 28, edited by D. O. Thompson and D. E. Chimenti (AIP, Melville, 2009), pp. 19-32.

\title{
NONDESTRUCTIVE EVALUATION OF METAL FATIGUE USING NONLINEAR ACOUSTICS
}

\author{
John H. Cantrell \\ NASA Langley Research Center, Mail Stop 231, Hampton, VA 23681, USA
}

\begin{abstract}
Safe-life and damage-tolerant design philosophies of high performance structures have driven the development of various methods to evaluate nondestructively the accumulation of damage in such structures resulting from cyclic loading. Although many techniques have proven useful, none has been able to provide an unambiguous, quantitative assessment of damage accumulation at each stage of fatigue from the virgin state to fracture. A method based on nonlinear acoustics is shown to provide such a means to assess the state of metal fatigue. The salient features of an analytical model are presented of the microelastic-plastic nonlinearities resulting from the interaction of an acoustic wave with fatigue-generated dislocation substructures and cracks that predictably evolve during the metal fatigue process. The interaction is quantified by the material (acoustic) nonlinearity parameter $\beta$ extracted from acoustic harmonic generation measurements. The $\beta$ parameters typically increase monotonically by several hundred percent over the fatigue life of the metal, thus providing a unique measure of the state of fatigue. Application of the model to aluminum alloy 2024-T4, 410Cb stainless steel, and IN100 nickel-base superalloy specimens fatigued using different loading conditions yields good agreement between theory and experiment. Application of the model and measurement technique to the on-site inspection of steam turbine blades is discussed.
\end{abstract}

Keywords: Nonlinear Acoustics, Metal Fatigue, Microelastic-Plastic Nonlinearity, Dislocation Substructures

PACS: $62.20 . \mathrm{Mk}, 61.72 . \mathrm{Hh}, 62.65 .+\mathrm{k}, 43.25 . \mathrm{Ba}$

\section{INTRODUCTION}

One of the longstanding goals of nondestructive evaluation has been to develop a quantitative means of assessing the state of metal fatigue and, hence, the remaining life of the material. Over the last several decades a number of techniques have been developed, but none of them has been able to provide a quantitative, unambiguous assessment of the state of fatigue from the virgin state to fracture. We show here that nonlinear acoustics offers great promise as a means of achieving this longstanding goal.

Nonlinear acoustics as a scientific discipline spans a range of physical phenomena, but we consider here only that phenomena associated with acoustic harmonic 
generation. The first experimental evidence of acoustic harmonic generation in solids was reported in 1963 independently by Breazeale and Thompson [1] and by Gedroits and Krasilnikov [2]. The harmonic generation in their studies resulted from elastic nonlinearity in their samples. In 1965 Hikata, Chick, and Elbaum [3] showed that one could also obtain considerable acoustic harmonic generation from large densities of single dislocations (dislocation monopoles) in materials. In 1994 Cantrell and Yost [4] showed that substantial acoustic harmonic generation could be obtained from dislocation dipoles generated during plastic deformation and fatigue. The first studies of harmonic generation from fatigued materials per se were reported by Yermilin et al. [5] in 1973 and by Buck [6] in 1976. The first analytical model of harmonic generation from cracks was reported by Richardson [7] in 1979.

Over the last several years bulk wave measurements of the nonlinearity parameter, a quantitative measure of acoustic harmonic generation, were reported by $\mathrm{Na}$, Cantrell, and Yost [8] for stainless steel, Frouin et al. [9] for Ti-6Al-4V, Cantrell and Yost [10] for AA2024, and Kim et al. [11] for IN100 nickel-base superalloy. The most significant findings of these four bulk wave studies are (1) the magnitude of the nonlinearity parameter increases monotonically for all materials measured as a function of percent full fatigue life from the virgin state to fracture (yielding a one-to-one relationship between the value of the nonlinearity parameter and the state of fatigue) and (2) the increase in the value of the nonlinearity parameter is typically of the order of several hundred percent.

In view of the findings of the four bulk wave studies, our research objectives over the last several years have been (1) to develop a physics-based model of the nonlinear interactions of acoustic waves with fatigue-generated substructures that predictably evolve during the fatigue process and (2) to compare model predictions to experimental measurements to establish the physical basis for developing a reliable fatigue-monitoring system using acoustic harmonic generation. We present a summary of the results obtained to date beginning with a discussion of acoustic harmonic generation from the most prominent sources of nonlinearity in fatigued metals along with their quantitative measures - the nonlinearity parameters.

\section{ACOUSTIC HARMONIC GENERATION IN FATIGUED METALS}

Let us consider launching a purely sinusoidal acoustic wave into a solid sample having parallel opposite faces by means of a narrowband transducer attached to one of the sample faces. As the wave propagates through the material, the wave will become distorted as the result of a number of sources of nonlinearity within the material. A Fourier transform of the signal received at the opposite face of the sample would reveal that the received signal is composed not only of the fundamental wave launched into the solid but also harmonic components of the fundamental wave as the result of waveform distortion generated by the various nonlinear sources within the material. One source of nonlinearity common to all materials is that from elastic nonlinearity (lattice anharmonicity). 


\section{Elastic Nonlinearity}

A quantitative measure of elastic nonlinearity may be obtained from the nonlinear Hooke's Law

$$
\sigma=A_{2}^{e}(\partial u / \partial a)+(1 / 2) A_{3}^{e}(\partial u / \partial a)^{2}+\cdots=A_{2}^{e}\left[(\partial u / \partial a)-(1 / 2) \beta^{e}(\partial u / \partial a)^{2}+\cdots\right]
$$

where $\sigma$ is the stress, $u$ is the particle displacement, $a$ is the spatial coordinate, and $A_{2}{ }^{\mathrm{e}}$ and $A_{3}{ }^{\mathrm{e}}$ are, respectively, the second and third-order Huang elastic coefficients. The parameter $\beta^{\mathrm{e}}$ is the elastic nonlinearity parameter defined from the last equality in Eq.(1) as

$$
\beta^{e}=-\left(A_{3}^{e} / A_{2}^{e}\right)
$$

A quantitative measurement of $\beta^{\mathrm{e}}$ may be obtained by substituting Eq.(1) into Newton's Law, $\rho_{0}\left(\partial^{2} u / \partial t^{2}\right)=\partial \sigma / \partial a$, where $\rho_{0}$ is the mass density and $t$ is time. We

obtain the nonlinear wave equation, $\partial^{2} u / \partial t^{2}=c^{2}\left[1-\beta^{e}(\partial u / \partial a)\right]\left(\partial^{2} u / \partial a^{2}\right)$. A solution to the nonlinear wave equation, assuming the input waveform $u=u_{1} \cos (\omega t)$ at $a=0$, is $u$ $=u_{0}+u_{1} \cos (\omega t)+u_{2} \sin 2(\omega t-k a)+\cdots$, where $u_{2}=(1 / 8) k^{2} u_{1}^{2} a \beta^{\mathrm{e}}$. We thus find from the last expression that the elastic nonlinearity parameter $\beta^{\mathrm{e}}$ can be determined from measurements of the fundamental and second-harmonic amplitudes $u_{1}$ and $u_{2}$, respectively, the distance of wave propagation into the material $a$, and the wave number $k$ as

$$
\beta^{e}=8 u_{2} /\left(k^{2} u_{1}^{2} a\right)
$$

To estimate the contribution of $\beta^{\mathrm{e}}$ to the variation in the nonlinearity parameter measured in fatigued metals, we write [12]

$$
\left(\Delta \beta^{e} / \beta^{e}\right) \approx\left[\left(A_{4}^{e} / A_{3}^{e}\right)-\left(A_{3}^{e} / A_{2}^{e}\right)+2\right]\left(\sigma_{1} / A_{2}^{e}\right)
$$

where $A_{4}{ }^{\mathrm{e}}$ is the fourth order Huang elastic coefficient and $\sigma_{1}$ is the internal or residual stress in the solid. For typical values of the Huang coefficients and maximum values of the internal stress resulting from plastic deformation and fatigue, $\left(\Delta \beta^{\mathrm{e}} / \beta^{\mathrm{e}}\right)$ is of the order of one percent or less. This value is orders of magnitude less than the variation of the nonlinearity parameters measured in fatigued metals. We must, therefore, consider other sources of nonlinearity in fatigued metals to explain the measured variation.

\section{Nonlinearity from Dislocation Monopoles}

Cyclic loading in metals continuously generates large numbers of dislocations (both edge and screw dislocations) throughout the fatigue life. Screw dislocations generally annihilate as the result of cross-slip and we are left with a preponderance of edge dislocations in fatigued metals. A typical edge dislocation is shown in Fig. 1 for a simple cubic lattice where the filled circles represent atomic sites. A positive edge dislocation may be viewed as being formed by inserting an extra half-plane of atoms into 
(a) Positive edge dislocation

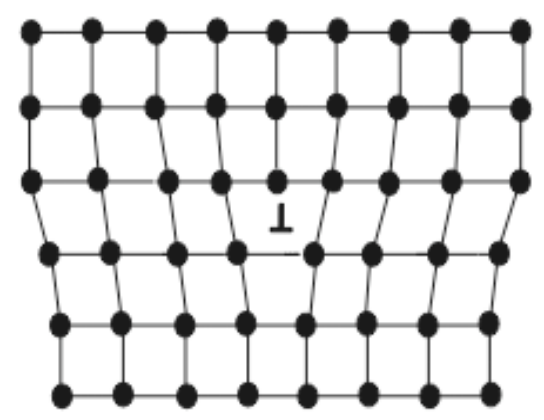

(b) Negative edge dislocation

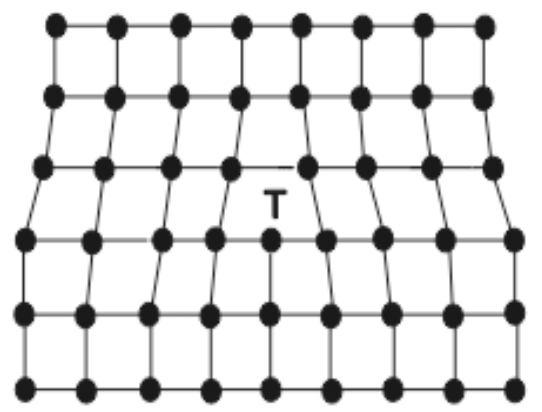

FIGURE 1. Edge dislocations in a simple cubic lattice.

the top half of the lattice as shown in Fig. 1a. The terminus of the extra half-plane of atoms defines the dislocation line and the symbol for a positive edge dislocation is the inverted "T". A negative edge dislocation, denoted by the upright " $T$ ", may be viewed as being formed by inserting the extra half-plane of atoms into the bottom half of the lattice as shown in Fig. $1 b$.

In 1965 Hikata, Chick, and Elbaum [3] developed a model for obtaining the nonlinearity parameter for single dislocations (monopoles) by assuming that the dislocations bow out into a smooth arc between two pinning points separated by the distance $2 L$ (the loop length) under the influence of an applied or internal stress $\sigma_{1}$. They obtain that the dislocation monopole nonlinearity parameter $\beta_{h c e}^{m p}$ is given by

$$
\beta_{h c e}^{m p}=\left[24 \Omega R^{3} \Lambda^{m p} L^{4}\left(A_{2}^{e}\right)^{2}\left|\sigma_{1}\right|\right] /\left(5 G^{3} b^{2}\right),
$$

where $\Lambda^{\mathrm{mp}}$ is the monopole dislocation density, $G$ is the shear modulus, $b$ is the magnitude of the Burgers vector, $\Omega$ and $R$ are strain and stress conversion factors, and $\left|\sigma_{1}\right|$ is the magnitude of the longitudinal internal stress at the site of the dislocation. Although the model of Hikata, Chick, and Elbaum has been used successfully for four decades, the model does not account for the effects of lattice resistive stresses on the bowing of the dislocations.

A more realistic model of dislocation bowing has been recently derived by Cantrell [13] by assuming that in moving under the influence of an applied or internal stress the dislocations encounter periodic lattice resistive shear stresses $\tau_{\text {lattice }}$ given by $\tau_{\text {lattice }}=-\tau_{P-N} \sin \left[2 \pi \xi(y) / a_{l s}\right]$, where $\tau_{\mathrm{P}-\mathrm{N}}$ is the Peierls-Nabarro barrier stress, $a_{\mathrm{ls}}$ is the lattice spacing, and $\xi(y)$ is the dislocation displacement. He obtains that the new dislocation monopole nonlinearity parameter $\beta^{\text {mp }}$ is given as [13] 
$\beta^{m p}=\beta_{h c e}^{m p}\left[1-\left(4 \tau_{P-N} / \pi R \sigma_{a m p l}\right) \sum_{n=1}^{\infty}(2 n-1)^{-1} J_{2 n-1}\left(2 \pi L^{2} R \sigma_{a m p l} / G b^{2}\right)\right]^{3}+\left(B_{1} / o_{a m p l}\right)^{6}$

where $\sigma_{\text {ampl }}$ is the stress amplitude of the acoustic wave launched into the material and $B_{1}$ is a constant. For large acoustic stress amplitudes Eq.(6) reduces to $\beta^{\mathrm{mp}} \approx \beta_{\text {hce }}{ }^{\mathrm{mp}}$, the results obtained by Hikata, Chick, and Elbaum [3]. However, at low stress amplitudes $\beta^{\mathrm{mp}}$ exhibits a Bessel function dependence on $\sigma_{\mathrm{ampl}}$. The damped oscillatory dependence of $\beta^{\mathrm{mp}}$ on $\sigma_{\mathrm{ampl}}$ predicted by Eq.(6) has been confirmed experimentally for single crystal Al[110] by Yost [14].

The damped oscillatory dependence of $\beta^{\mathrm{mp}}$ on $\sigma_{\mathrm{ampl}}$ disappears when Eq.(6) is applied to polycrystalline solids with randomly oriented grains. However, a strong hooklike behavior occurs at very low stress wave amplitudes. The hook-like behavior has been reported by Barnard [15] who obtained experimental measurements of the nonlinearity parameters of four polycrystalline $\mathrm{Cu}-\mathrm{Al}$ alloys as a function of the wave displacement amplitude (linearly proportional to the stress amplitude). The experimental results of Barnard [15] are shown in Fig. 2. The hook-like behavior is often called the Buck hook in honor of the late Otto Buck. We see here that the Buck hook is a real phenomenon associated with dislocation dynamics at low acoustic drive amplitudes.

\section{Nonlinearity from Dislocation Dipoles}

A third source of nonlinearity in fatigued materials is obtained by noting that cyclic loading forces the dislocations to move to-and-fro along their glide paths denoted

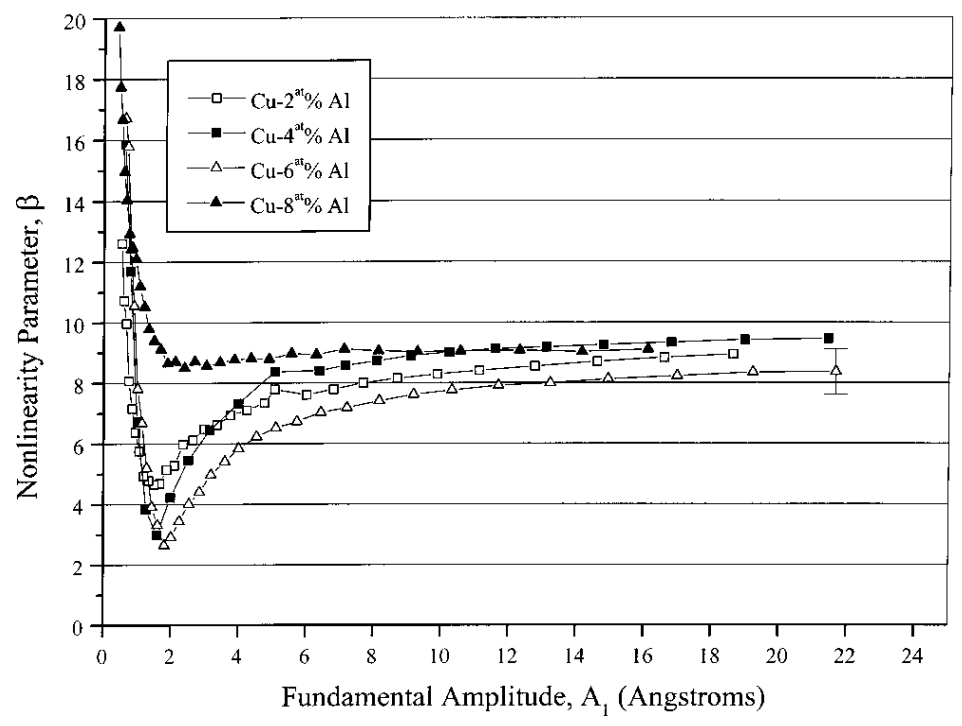

FIGURE 2. Graph of nonlinearity parameters of four $\mathrm{Cu}-\mathrm{Al}$ alloys plotted as a function of wave displacement amplitude (linearly proportional to stress amplitude $\sigma_{\text {ampl }}$ ) (from ref. 15). 


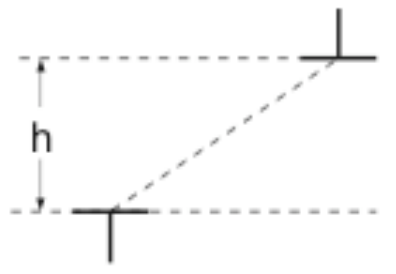

FIGURE 3. Dislocation dipole with dipole height $h$.

by the horizontal dotted lines in Fig. 3. When two dislocation of opposite polarity move sufficiently close to each other, they become trapped in each other's stress field and form a stable dislocation pair known as a dislocation dipole. The distance between the glide planes of the dipole is known as the dipole height $h$. Cantrell [12] has shown that the dislocation dipole nonlinearity parameter is obtained as

$$
\beta^{d p}=\left[16 \pi^{2} \Omega R^{2} \Lambda^{d p} h^{3}(1-v)^{2}\left(A_{2}^{e}\right)^{2}\right] /\left(G^{2} b\right)
$$

where $\Lambda^{\mathrm{dp}}$ is the dipole density and $v$ is the Poisson ratio. We note that the magnitude of the internal stress at the site of the dislocation does not enter into Eq.(7), unlike the equation for the monopole nonlinearity parameter.

\section{SELF-ORGANIZATION OF DISLOCATION SUBSTRUCTURES}

As fatigue continues, the increasing numbers of dislocation dipoles lead to a selforganization of the dipoles into specific substructures that depend on the slip character of the material. The slip character is determined by several material properties including the stacking fault energy, the magnitude of the shear modulus, the magnitude of the Burgers vector, and the short-range order in the material. In metals there are two distinct slip characters - wavy slip and planar slip. We first consider wavy slip metals.

\section{Wavy Slip Dislocation Substructures}

The first substructure into which dipoles organize in wavy slip metals is the vein structure illustrated in Fig. 4. Fig. 4a is a schematic of the cross-section of the vein

(a)

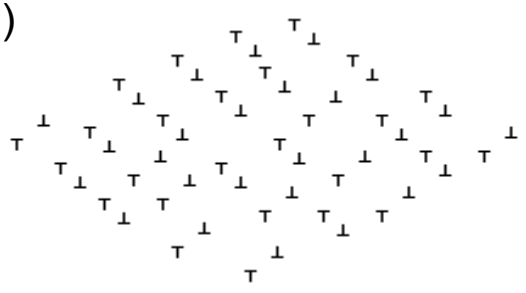

(b)

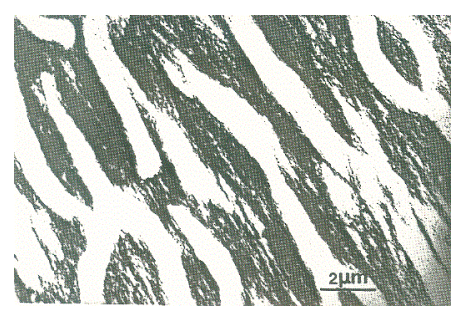

FIGURE 4. Vein structure in wavy slip metals: (a) Schematic of vein cross section. (b) TEM micrograph (longitudinal view) (from ref. 16). 
(a)

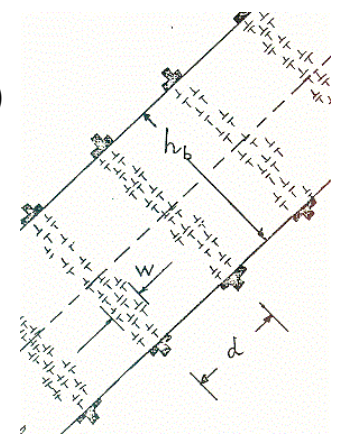

(b)

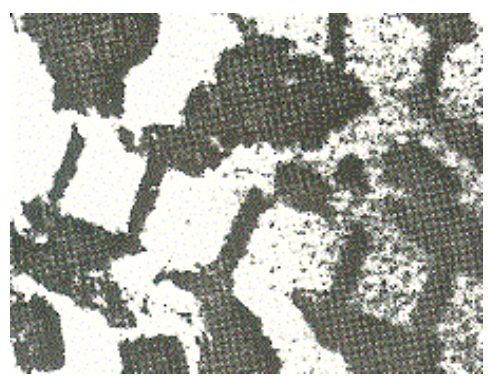

FIGURE 5. PSB structure in wavy slip metals: (a) Schematic of dipole arrangements (from ref. 16). (b) TEM micrograph (from ref. 16).

structure. We see that the vein structure consists primarily of a dense array of dipoles both of the interstitial type (extra half-plane of each dislocation intersects the glide plane of the other dislocation) and the vacancy type (extra half-plane of each dislocation does not intersect the glide plane of the other). Fig. 4b shows a transmission electron micrograph of vein structure in single crystal $\mathrm{Cu}$ (longitudinal view) obtained by Jin [16]. The dark regions are the vein structures. The light regions between the vein structures are relatively dislocation-free regions called channels. For metals with high stacking fault energies such as aluminum and many aluminum alloys the "vein" or matrix structure is cellular rather than vein-like.

With further cyclic loading both the volume fraction of vein structure and the dislocation density in the veins increase until a critical value of the dislocation density is attained that produces an elastic instability in the vein structure. The elastic instability drives the transformation of the vein structure into the more stable persistent slip band (PSB) structure shown in Fig. 5. Fig. 5a is a schematic of the PSB structure. It is seen that the PSB has a ladder-like morphology with the rungs (also called hedges) of the ladder consisting of linear arrays of dipoles. Fig. 5b shows a transmission electron micrograph of PSBs in single crystal Cu obtained by Jin [16]. The hedges are the dark rungs on the ladder and the light regions between the hedges are channel regions consisting of dislocation monopoles roughly two order of magnitude less dense than the dislocations in the hedges.

We conclude that dislocation dipoles and monopoles are the basic building blocks of the veins and persistent slip bands generated during fatigue of wavy slip metals. The dislocation dipoles account for the preponderance of dislocation configurations generated during fatigue. The dislocation monopoles dominate the channel regions of the PSBs and account for the observed large plastic strains produced in PSBs under load.

\section{Planar Slip Dislocation Substructures}

The first dislocation substructures generated in planar slip metals consist of dislocations arranged in thin, linear bands known as planar slip bands. Fig. 6a is a schematic of a planar slip band. We see that the planar slip bands are composed of linear arrays of dislocation dipoles, as well as monopoles. Fig. 6b shows a transmission electron micrograph of planar slip bands in $\alpha$-brass obtained by Lukás and Klesnil [17]. 
(a)

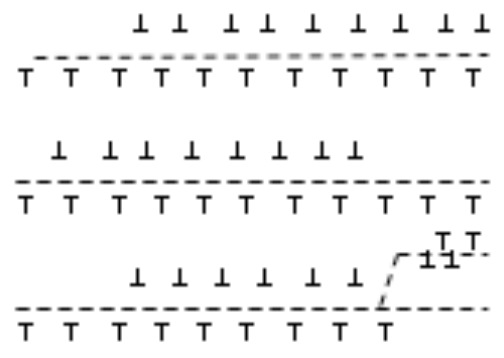

(b)

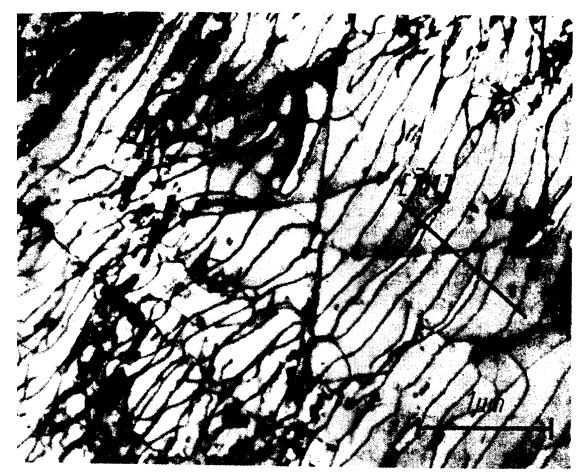

FIGURE 6. Planar slip bands and persistent Luders (PLBs) bands in planar slip metals: (a) Schematic of planar slip bands. (b). TEM micrograph showing planar slip bands (long thin lines) and PLBs (large, dark patches) in $\alpha$-brass (from ref. 17).

The dark, thin lines are the planar slip bands. The large, dark regions in Fig. $6 \mathrm{~b}$ are dense arrays of planar slip bands known as persistent Luders bands (PLBs). The PLBs are somewhat analogous evolutionarily to the PSBs of wavy slip metals and the persistent slip bands are somewhat analogous to the vein structure of wavy slip metals.

\section{SUBSTRUCTURAL EVOLUTION, CRACK PROPAGATION, AND RELATION TO NONLINEARITY PARAMETERS IN WAVY SLIP METALS}

\section{Elastic-Plastic Nonlinearity}

We have established that the basic building blocks of the veins and PSBs generated in wavy slip metals during fatigue are dislocation dipoles and monopoles. Although dipoles and monopoles also serve as basic building blocks of the planar slip bands and PLBs generated in planar slip metals, we postpone discussion of planar slip metals until we consider harmonic generation in wavy slip metals in more detail.

We have identified three sources of harmonic generation in fatigued wavy slip metals and three corresponding nonlinearity parameters - the elastic nonlinearity parameter $\beta^{\mathrm{e}}$ and two plastic ones, the monopole nonlinearity parameter $\beta^{\mathrm{mp}}$ and the dipole nonlinearity parameter $\beta^{\mathrm{dp}}$. Contrary to what one might expect, however, the total elastic-plastic nonlinearity parameter $\beta^{\mathrm{e}-\mathrm{p}}$ is not equal to the simple sum of $\beta^{\mathrm{e}}, \beta^{\mathrm{mp}}$, and $\beta^{\mathrm{dp}}$, but rather is given by [12]

$$
\beta^{e-p}=\frac{\beta^{e}+f^{m p} \beta^{m p}+f^{d p} \beta^{d p}}{\left(1+f^{m p} \Gamma^{m p}+f^{d p} \Gamma^{d p}\right)^{2}}
$$

where $f^{\mathrm{mp}}$ is the volume fraction of veins and PSBs containing monopoles, $f^{\mathrm{dp}}$ is the volume fraction of veins and PSBs containing dipoles, 
and

$$
\Gamma^{m p}=(2 / 3)\left(\Omega R \Lambda^{m p} L^{2} A_{2}^{e} / G\right)
$$

$$
\Gamma^{d p}=\left(4 \pi A_{2}^{e} \Omega R \Lambda^{d p} h^{2}(1-v) / G\right) .
$$

It is important to note that $f^{\mathrm{mp}}$ and $f^{\mathrm{dp}}$ increase monotonically with advancing states of fatigue and make the dominant contributions to the variation in the elastic-plastic nonlinearity parameter as a function of percent fatigue life.

Eq.(8) was derived under the assumption that the fatigue damage is distributed uniformly throughout the material. However, the fatigue damage is generally quite localized. Let $V_{\mathrm{W}}$ represent the volume of material swept out by the ultrasonic wave in passing through the material and let $V_{\mathrm{F}}$ represent that portion of the total volume of fatigue damage contained in $V_{\mathrm{W}}$. We define the wave interaction factor $f_{\mathrm{WI}}$ as $\mathrm{f}_{\mathrm{WI}}=$ $V_{\mathrm{F}} / V_{\mathrm{W}}$. Using this definition of the wave interaction factor, Cantrell [18] has shown that the effective elastic-plastic nonlinearity parameter for materials with localized fatigue damage is more appropriately given by

$$
\beta^{e-p}=\frac{\beta^{e}+f_{W I}\left(f^{m p} \beta^{m p}+f^{d p} \beta^{d p}\right)}{\left[1+f_{W I}\left(f^{m p} \beta^{m p}+f^{d p} \beta^{d p}\right)\right]^{2}}
$$

The wave interaction factor can be calculated as [18]

$$
f_{W I}=\left(f^{m p} \Gamma^{m p}+f^{d p} \Gamma^{d p}\right)^{-1}\left[\left(c_{0} / c_{F}\right)^{2}-1\right]
$$

where $c_{0}$ is the phase velocity measured in the virgin state and $c_{\mathrm{F}}$ is the phase velocity measured in the fatigue state. The calculations for $f_{\mathrm{WI}}$ become more accurate at the advanced states of fatigue as the dislocation substructures begin to dominate sample inhomogeneity with regard to measurements of $c_{\mathrm{F}}$. We also note that $f_{\mathrm{WI}}$ serves as a scaling or normalizing factor that somewhat adjusts for errors in assigning values to the dislocation density, loop length, and dipole height.

\section{Crack Nonlinearity}

A fourth source of nonlinearity in fatigued metals is that of crack initiation and growth. Crack initiation generally occurs upon formation of persistent slip bands in wavy slip metals and can occur as early as $0.1 \%$ of the total fatigue life. However, the cracks do not become measurable using bulk wave harmonic generation until the cracks have grown to a size where the Paris-Erdogan Law applies (typically 80-95\% fatigue life for high cycle fatigue). Cantrell [19] has applied the Paris-Erdogan Law [20] of crack propagation to the Nazarov-Sutin [21] crack nonlinearity model to obtain the crack growth nonlinearity parameter $\beta^{\text {crk }}$ as

$$
\beta^{c r k}=\frac{5.3 \times 10^{6} C_{c r k}}{\left[a_{1}^{n-2}+a_{2}(1-f)\right]^{8 /(n-2)}}\left[1+\frac{0.25 C_{c r k}}{\left[a_{1}^{n-2}+a_{2}(1-f)\right]^{6 /(n-2)}}\right]
$$

where 


$$
a_{1}=B \sigma_{\max } / K_{1 c} \quad, \quad a_{2}=(n-2) N_{\text {total }} A(B \Delta \sigma)^{n} / 2,
$$

$C_{\text {crk }}$ is the crack concentration, $N_{\text {total }}$ is the number of fatigue cycles to failure, $K_{1 \mathrm{c}}$ is the fracture toughness, $f$ is the fraction of fatigue life, $\Delta \sigma$ is the applied stress range, $B$ is the crack geometry factor, and $A$ and $n$ are experimentally determined material-dependent parameters.

\section{$\underline{\text { Total Nonlinearity Parameter }}$}

The total nonlinearity parameter $\beta^{\text {total }}$ is the sum of the elastic-plastic nonlinearity parameter and the crack growth nonlinearity parameter

$$
\beta^{\text {total }}=\beta^{e=p}+\beta^{c r k} \quad .
$$

However, before applying the model equations to progressive fatigue damage it is essential to have a detailed knowledge of the volume fractions of veins and persistent slip bands generated in wavy slip metals as a function of the percent fatigue life of the metal. This knowledge allows the calculation of the volume fractions of dislocation monopoles $f^{\mathrm{mp}}$ and dipoles $f^{\mathrm{dp}}$ that appear directly in the model equations.

Grobstein et al. [22] have published extensive data on the evolution of veins and persistent slip bands from the virgin state to near fracture on specimens of polycrystalline pure Ni subjected to a variety of loading conditions. However, similarly comprehensive data for other metals are not generally available in the literature. We thus make the critical assumption that the evolution of veins and persistent slip bands obtained for prototypical wavy slip pure $\mathrm{Ni}$ is representative of all wavy slip metals with appropriate adjustments to account for the effects of alloying elements and microstructure. This assumption has been tested on two wavy slip alloys.

\section{APPLICATION OF MODEL TO FATIGUED METALS}

Fatigue experiments were performed on specimens of aluminum alloy 2024-T4 and $410 \mathrm{Cb}$ stainless steel using ASTM standard cylindrical "dog-bone" specimens fatigue in uniaxial, stress-controlled loading with an R-value (minimum load/maximum load) of zero. Harmonic generation measurements were taken on samples cut from the gauge section of the specimens after fatiguing. Different specimens were used for each targeted number of fatigue cycles.

A plot of the nonlinearity parameter as a function of the percent full fatigue life of AA2024-T4 (276MPa loading) is shown in Fig. 7. The solid line is the theoretical curve obtained from the model equations, using the substructural evolutionary data of Grobstein et al. [22]. The filled circles are the experimental data points measured by moving the transducer to different positions on the samples to obtain the maximum values of the nonlinearity parameter. The agreement between theory and experiment is excellent.

A plot of the nonlinearity parameter as a function of the percent full fatigue life of $410 \mathrm{Cb}$ stainless steel is shown in Fig. 8. Again, the solid line is the theoretical curve obtained from the model equations, using the substructural evolutionary data of Grobstein 


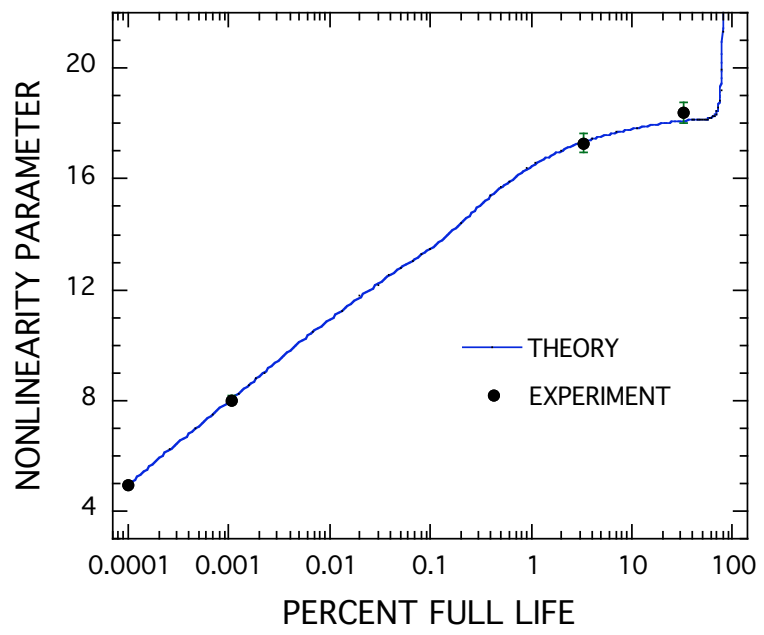

FIGURE 7. Graph of nonlinearity parameter of aluminum alloy 2024-T4 fatigued in stress-controlled loading at 276MPa plotted as a function of percent full fatigue life.

et al. [22]. The filled circles are the experimental data points. We note that there is considerably more scatter in the data points than was obtained for AA2024-T4. This is in large part due to the fact that the transducer was not moved to various positions on the sample surface to obtain the maximum values of $\beta^{\text {total }}$. All measurements were taken along the cylindrical axes of the samples. Nonetheless, the overall agreement between theory and experiment is good.

Following the data taken on $410 \mathrm{Cb}$ stainless steel, a beta (nonlinearity parameter) scan was performed on a retired-for-cause steam turbine blade (also made of $410 \mathrm{Cb}$

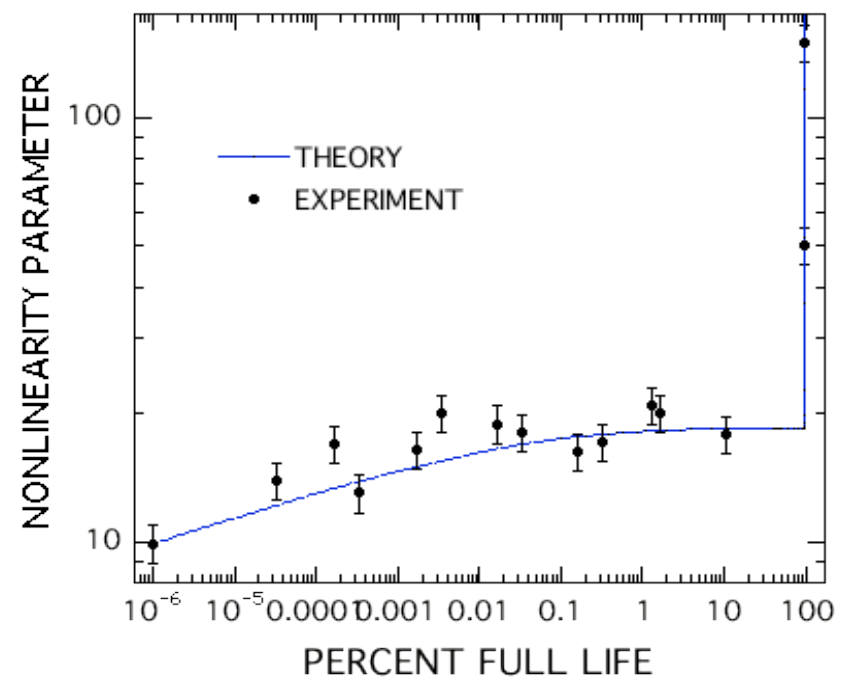

FIGURE 8. Graph of nonlinearity parameter of $410 \mathrm{Cb}$ stainless steel fatigued in stress-controlled loading at 551MPa plotted as a function of percent full fatigue life. 


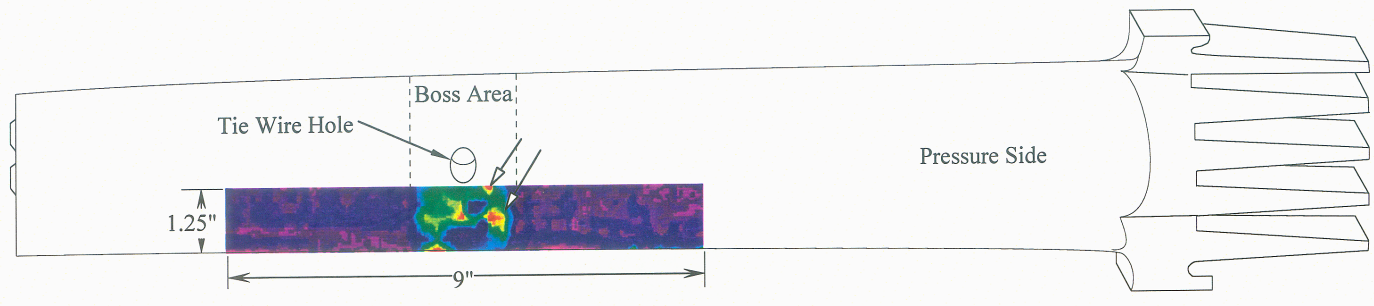

FIGURE 9. Beta scan of retired-for-cause steam turbine blade showing regions of critical fatigue damage where $\beta>135$.

stainless steel) obtained from Dominion Power Corporation. The scan, shown in Fig. 9, was performed in the "boss" area of the blade and revealed regions of critical fatigue damage where the nonlinearity parameter was measured to be greater than 135 . Following the beta scan, beta measurements were taken during an on-site inspection at one of Dominion Power Corporation's electrical generating facilities. A $\beta$-value of roughly 150 , indicating critical fatigue damage, was measured for one of the turbine blades. However, the blade was not replaced, because none of the other NDI techniques revealed such critical damage. The catastrophic failure of that blade several months later resulted in major damage to the turbine housing. Dominion Power now uses beta measurements routinely as part of their inspection toolkit.

\section{NONLINEARITY PARAMETERS FOR PLANAR SLIP METALS}

It was pointed out in the above discussion on the substructural evolution of planar slip metals that the persistent Luders bands (PLBs) are somewhat analogous evolutionarily to the persistent slip bands (PSBs) of wavy slip metals and that the persistent slip bands are somewhat analogous to the vein structure of wavy slip metals. More importantly, both the PLBs and the planar slip bands are composed of dislocation dipoles and monopoles that serve as basic building blocks of the planar slip substructures. This suggests that the nonlinearity parameter model developed for wavy slip metals may in fact apply to planar slip metals as well. Further evidence that this may be the case is furnished by Hong and Laird [23] who show that for the planar slip $\mathrm{Cu}-\mathrm{Al}$ alloys composed of wavy slip Cu base metal the total fatigue lives are related as $N_{\mathrm{PS}}=N_{\mathrm{WS}} / f_{\mathrm{PSB}}$ where $N_{\mathrm{PS}}$ is the number of cycles to failure for the planar slip alloy, $N_{\mathrm{WS}}$ is the number of cycles to failure for the wavy slip metal of which the planar slip alloy is composed, and $f_{\mathrm{PSB}}$ is the volume fraction of PSBs for the base metal at failure.

Encouraged by these results, we have applied the model developed for wavy slip metals to planar slip IN100 nickel-base superalloy [24]. The results are shown in Fig. 10 where the normalized nonlinearity parameter is plotted as a function of the percent full fatigue life of the metal. The solid line is the theoretical curve obtained from the 


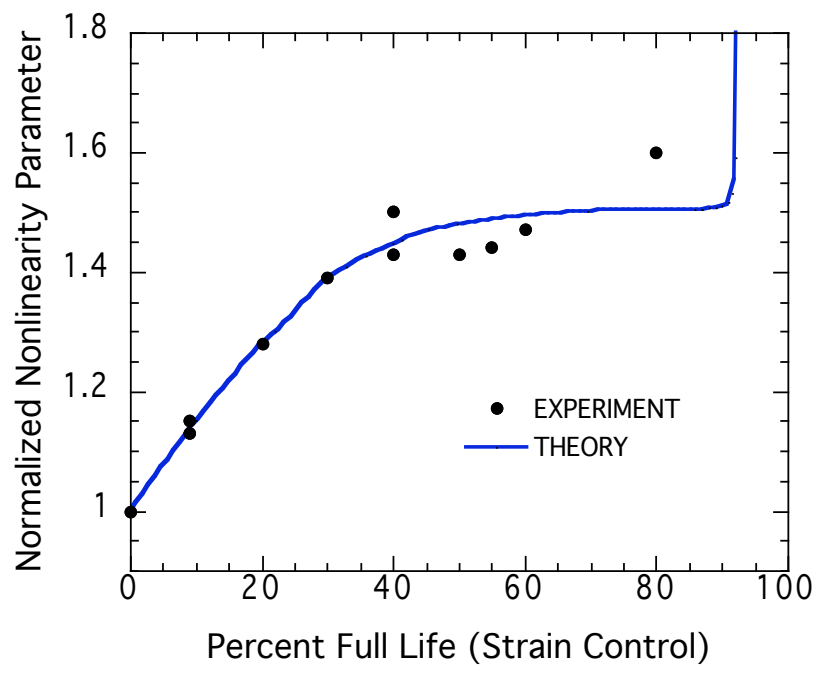

FIGURE 10. Graph of nonlinearity parameter of IN100 fatigued in strain-controlled loading at $0.48 \%$ plotted as a function of percent full fatigue life.

present model and the filled circles are experimental data points taken from the paper by Kim et al. [11]. Again, the agreement between theory and experiment is quite good and indicates that the present model, although originally developed for wavy slip metals, may in fact be applicable to a wider variety of metals than originally intended. The wider application may be due to the somewhat generic nature of the model in relating the nonlinearity parameters to the fundamental dislocation dipole and monopole building blocks of the evolving fatigue-generated substructures.

\section{SURFACE ACOUSTIC WAVE MEASUREMENTS}

Since most fatigue damage is manifested near the surface of materials, it is often assumed that surface acoustic wave (SAW) measurements of the nonlinearity parameters would provide the most expedient and efficient means of assessing accumulated fatigue damage. However, critical fatigue damage is not always first manifested at the surface. In many cases, especially for "dirty" alloys, critical damage occurs in the bulk of the material at inclusions, grain boundaries, and at interior cracks produced in the bulk during materials processing. For such cases SAW measurements would not detect such critical damage.

Additionally, the appearance of linear arrays of cracks along the surface wave propagation path affects the SAW $\beta$ measurements differently for wavy slip and planar slip metals. For planar slip metals the cracks initiate late in the fatigue life, generally only after the PLBs completely fill the gauge section of the specimen during cyclic loading. In such cases the evolution of the dislocation substructures dominates the $\beta$ measurements. Such a situation has been shown by Herrmann et al. [25] in a plot of both 
bulk and SAW measurements of $\beta$ as a function of percent fatigue life of IN100 nickelbase superalloy. The bulk and SAW measurements are in agreement until roughly $85 \%$ of the fatigue life, where the $\beta$-value obtained from SAW measurements is dramatically reduced.

For wavy slip metals microcracks initiate soon after the appearance of PSBs (as early as $0.1 \%$ of the fatigue) and make significant contributions to SAW measurements for the remainder of the fatigue life. The contributions are documented by Ogi, Hirao, and Aoki [26] who plot the second harmonic amplitude divided by the fundamental amplitude as a function of percent fatigue life for wavy slip low carbon steel. They obtain two peaks in the curve at roughly $60 \%$ and $85 \%$ of the fatigue life as the result of cracks interacting with the fatigue-generated dislocation substructures. The peaks introduce an ambiguity in the assessment of the state of fatigue by destroying the one-toone relationship between the value of the nonlinearity parameter and the percent full fatigue life of the material.

\section{CONCLUSION}

In accordance with the research objectives stated in the introduction we (1) have developed an experimentally validated physics-based model of the nonlinearity parameter as a function of the percent fatigue life of the metal and (2) have applied the model to develop a successful fatigue monitoring system as demonstrated during field inspections at Dominion Power Corporation. But the research is far from complete. Future research must necessarily focus on developing new, user-friendly, sensor technologies that allow measurements in geometrically confined/constrained environments. In addition, it is essential to continue to establish the scientific underpinnings for the emerging technologies via model improvements and experimental measurements for a variety of metals that also includes the influences of the environment, including environmentallydriven material degradation, on the nonlinearity parameters.

\section{REFERENCES}

1. M. A. Breazeale and D. O. Thompson, Appl. Phys. Lett. 3, 77-78 (1963).

2. A. Gedroits and V. A. Krasilnikov, Sov. Phys. - JETP 16, 1122-1126 (1963).

3. A. Hikata, B. B. Chick, and C. Elbaum, J. Appl. Phys. 36, 229-236 (1965).

4. J. H. Cantrell and W. T. Yost, Philos. Mag. 69, 315-326 (1994).

5. J. V. Yermilin, L. K. Zarembo, V. A. Krasilnikov, Ye. D. Mezintsev, V. M. Prokhorov, and K. V. Khilkov, Phys. Met. Metallogr. 36, 174 (1973).

6. O. Buck, IEEE Trans. Son. Ultrason. 23, 346 (1976).

7. J. M. Richardson, Int. J. Eng. Sci. 17, 73-85 (1979).

8. J. K. Na, J. H. Cantrell, and W. T. Yost, in Review of Progress in Quantitative Nondestructive Evaluation, 15B, edited by D. O. Thompson and D. E. Chimenti, Plenum, New York, 1996, pp. 1347-1352.

9. J. Frouin, S. Sathish, T. E. Matikas, and J. K. Na, J. Mater. Res. 14, 1295-1298 (1999).

10. J. H. Cantrell and W. T. Yost, Int. J. Fatigue 23, S487-S490 (2001). 
11. J.-Y. Kim, L. J. Jacobs, J. Qu, and J. W. Littles, J. Acoust. Soc. Am. 120, 1266-1273 (2006).

12. J. H. Cantrell, Proc. R. Soc. London A 460, 757-779 (2004).

13. J. H. Cantrell, (to be published).

14. W. T. Yost, (to be published).

15. D. J. Barnard, Appl. Phys. Lett. 74, 2447-2449 (1999).

16. N. Y. Jin, Ph.D. thesis, University of Cambridge, 1985.

17. P. Lukás and M. Klesnil, Phys. Stat. Solidi 37, 833-842 (1970)

18. J. H. Cantrell, J. Appl. Phys. 100, 063508 (2006).

19. J. H. Cantrell, Philos. Mag. 86, 1539-1554 (2006).

20. P. C. Paris and F. Erdogan, Trans. ASME J. Basic Eng. 85, 528 (1963).

21. V. E. Nazarov and A. M. Sutin, J. Acoust. Soc. Am. 102, 3349-3354 (1997).

22. T. L. Grobstein, S. Sivashankaran, G. Welsch, N. Panigrahi, J. D. McGervey, and J. W. Blue, Mater. Sci, Eng. A 138, 191-203 (1991).

23. S. I. Hong and C. Laird, Fatigue Fract. Engng. Mater. Struct. 14, 143-169 (1991).

24. J. H. Cantrell, (to be published).

25. J. Herrmann, J.-Y. Kim, L. J. Jacobs, J. Qu, J. W. Littles, and M. F. Savage, J. Appl. Phys. 99, 12913 (2006)

26. H. Ogi, M. Hirao, and S. Aoki, J. Appl. Phys. 90, 438-442 (2001). 